\title{
Relic Entropy Generation, Relic Gravitons and Relic Neutrinos, and Interaction of Both in CMBR
}

\author{
A.W.Beckwith \\ Chongqing University Department of Physics: Chongqing University, Chongqing, PRC , 400044 \\ Corresponding Author: abeckwith@uh.edu
}

Copyright $@ 2013$ Horizon Research Publishing All rights reserved.

\begin{abstract}
First of all, we outline a well known result. I.e. the formation of energy density and energy flux density for de Sitter space time, assuming close to monochromatic close to plane wave generation of spin two gravitons. This is done in the context of an emergent vacuum energy field being introduced at the onset of the initial space time singularity. The end result of our discussion is to purport another explanation of the gravitational lensing and Neutrino physics turbulent behavior as presented to the Author in QuyNohn, by Dr. Turner, in the Windows to the Universe ICISE inaugural conference 2013.
\end{abstract}

Keywords Vacuum Energy; Entropy, Brane- Anti Brane, Neutrinos

\section{Introduction: Relic Perturbations of De Sitter Space Time, and its Links to Creating Energy Density and Energy Flux Density}

We are looking first of all at a classical treatment of spin two gravitons due to GR field equations, using the datum that in most situation we have near Euclidian flat space for reasons we will detail in an appendix entry. The starting point to all of this is to look at the line element of $d S^{2}=g_{\mu \nu} d x^{\mu} d x^{v}$ for a spin two graviton influenced value of a metric with $a$ as the scaled expansion 'distance' and $h_{\oplus}$ and $h_{\circledast}$ as spin two graviton "particles" so the metric looks like

$$
g_{\mu v}=\left[\begin{array}{cccc}
-a^{2} & 0 & 0 & 0 \\
0 & a^{2} \cdot\left(1+h_{\oplus}\right) & a^{2} h_{\otimes} & \\
0 & a^{2} h_{\otimes} & a^{2} \cdot\left(1-h_{\oplus}\right) & \\
0 & 0 & 0 & a^{2}
\end{array}\right]
$$

This leads to a 'single' monochromatic wave representation in an arbitrary $z$ direction write up of the energy density and the energy flux density looking like, assuming the Einstein field equations, leading to a spin two 'graviton' energy density

$$
t_{0}^{0}=\frac{c^{4} k^{2}}{4 \pi G a^{2}} \cdot\left(\mathrm{h}_{\oplus}^{2}+h_{\circledast}^{2}\right)
$$

Buonanno uses a plane wave, TT gauge treatment of gravity wave of stress energy tensor with [2]

$$
t_{00}=\frac{c^{2}}{16 \pi G}\left\langle\dot{h}_{\otimes}^{2}+\dot{h}_{\oplus}^{2}\right\rangle \equiv \frac{c^{2}}{32 \pi G}\left\langle\dot{h}_{i j}^{T T} \dot{h}_{i j}^{T T}\right\rangle
$$

So Buonanno gets a value of gravitational wave energy flux per unit area of this as [2]

$$
\frac{d E}{d t d A}=\frac{c^{3}}{16 \pi G}\left\langle\dot{h}_{\otimes}^{2}+\dot{h}_{\oplus}^{2}\right\rangle \sim \frac{c^{3} f^{2} h^{2}}{16 \pi G}
$$

The important scaling of Eqn. 2ab which we can reference here for future inquiry is that allegedly, according to Buonanno, [1],[2] the following table1 can be made as to for the values given in $\operatorname{Eq}(2 \mathrm{ab})$ for supernova gravity wave bursts, neutrino energy flux, and photon energy flux , along the lines of wave energy flux per unit area for a super novae

Table 1. Order of magnitude comparison of signal gravitational wave energy flux per unit area due to data as presented in [1] and [2]

\begin{tabular}{|c|c|c|c|}
\hline & $\begin{array}{c}\text { A few mili } \\
\text { seconds }\end{array}$ & A few seconds & One week \\
\hline $\mathrm{GW}$ & $\begin{array}{c}400 \mathrm{erg} / \mathrm{cm}^{\wedge} 2 \\
\mathrm{sec}\end{array}$ & & \\
\hline $\begin{array}{c}\text { Neutrino } \\
\text { Energy flux }\end{array}$ & & $\begin{array}{c}10^{\wedge} 5 \mathrm{erg} / \\
\mathrm{cm}^{\wedge} 2 \mathrm{sec}\end{array}$ & \\
\hline $\begin{array}{c}\text { Optical } \\
\text { radiation }\end{array}$ & & & $\begin{array}{c}10^{\wedge}-4 \mathrm{erg} / \\
\mathrm{cm}^{\wedge} 2 \mathrm{sec}\end{array}$ \\
\hline
\end{tabular}

The implications of tablel as to relic gravitational waves will be elaborated upon in the later part of this paper. But it ties in with the supposition that there can and will be a linkage between data which can be obtained at Icecube as far as neutrinos, and relic gravitational waves. And then a 'graviton' energy flux density

$$
c \cdot t_{0}^{3}=\frac{i c^{5} k^{1}}{4 \pi G \stackrel{3}{a}} \cdot\left\{\dot{a} \cdot\left(\mathrm{h}_{\oplus}^{2}+h_{\otimes}^{2}\right)+\right.
$$




$$
\left.a \cdot\left(\mathrm{h}_{\oplus} \dot{\mathrm{h}}_{\oplus}+\mathrm{h}_{\otimes} \dot{\mathrm{h}}_{\otimes}\right)\right\}
$$

In the onset of inflation, we are able to make the following early universe approximation, that of $a \approx a_{\text {init }} \cdot \exp \left[H_{\text {init }} \cdot t\right]$ with $\quad a_{\text {init }} \sim \varepsilon<<1 \quad$ in magnitude so that if we make the additional quasi planar approximations, namely using $\frac{\partial}{\partial t} \rightarrow \mp i \omega_{g}$ as well as $\nabla \rightarrow i k_{g}$ for non trivial distance traveled (not a Plank length short distance), as well as a scalar wave magnitude approximation of

$$
A_{\oplus} \sim A_{\otimes} \approx A\left(k_{g}\right) / a(t)
$$

So we then get

$$
\begin{array}{r}
h_{\oplus} \cong A_{\oplus} \mathrm{e} \times\left[\dot{\mathrm{p}}\left(k_{g} z-\omega_{g} t\right)\right] \approx \\
A_{\otimes} \mathrm{e} \times\left[i \mathrm{\rho}\left(k_{g} z-\omega_{g} t\right)\right] \cong h_{\otimes}
\end{array}
$$

This will lead to the following value of the energy flux density, i.e.

$$
c \cdot t_{0}^{3}=\frac{i c^{5} k^{1} h_{\otimes}^{2}}{2 \pi G a^{2}} \cdot\left\{\omega_{g}+i H_{i n i t}\right\}+\text { H.O.T. }
$$

If we have large values of the gravitational wave frequency then of course we have a domination of equation six so that

$$
\left|c \cdot t_{0}^{3}\right|=\frac{c^{5} k^{1} h_{\circledast}^{2}}{2 \pi G a^{2}} \cdot\left\{\omega_{g}\right\}+\text { H.O.T. }
$$

For small values of gravitational wave frequency we have that in this case we will observe

$$
\left|c \cdot t_{0}^{3}\right|=\frac{c^{5} k^{1} h_{\otimes}^{2}}{2 \pi G a^{2}} \cdot\left\{H_{\text {init }}\right\}+\text { H.O.T. }
$$

When we examine the gravitational wave frequency, as contributing to energy flux density, as given by Eq. (6) to Eq. (8) we have a ready made energy flux, the wavelength of Gravitons, and then also the frequency of Gravitons to contend with. This if we then go to look at what several people, with $\mathrm{Ng}$ proposed[3], as given by also [4] in that

$$
S \sim n
$$

This can be then correlated, in part to presentations as to Entropic Gravity, also given by $\mathrm{Ng}$ [5] . As to what we are then for this problem relating the Graviton count, as given by a number, $n$, as in Eq. (9), and by infinite Quantum statistics relate it entropy via Eq.(9). In doing so we have to consider now how the entropy as by Eq. (9) may relate to energy flux as given by Eq. (7) above, and what it will say about the CMBR, later on. Not that the classical entropy formula as given below, if the same as Eq.(9) above, will through its temperature and volume, say something profound. Also we should keep in mind the subtle point raised by Eq. (7) of [6] by the author namely that, for the numerical count, as in Eq
(7) of reference [6] we have that if, then over a long period of time, we have, if $\mathrm{T}$ is temperature and $\Delta S$ a change in entropy over a long period of time as

$$
T \cdot \Delta S / \text { Dist } \rightarrow n
$$

If we look at Eq. (7) above, also, and also use Dist $\sim \lambda \sim 1 / \omega$, with the frequency also tied in with Eq. (7), we get then a specific relationship as to looking at an inter relationship of $\Delta S$, change of entropy, with a gravitational wave frequency, $\omega$, and a baseline temperature $\mathrm{T}$, as related to a graviton count, $\mathrm{n}$. This will then have some real implications as to the entropy developed classically which we comment upon below. Note that [7] says a lot more about the full form of Eq.(10) before time evolution lead to the evolution of it to in a limiting process, approaching a graviton count, $\mathrm{n}$.

\section{Introduction: Semi Classical Models of Entropy Compared with the Entropy quantum infinite statistics counting given by $\mathrm{Ng}$}

Kolb and Turner [8] have a temperature $T$ related entropy density which leads to that we are able to state total entropy as the entropy density time's space time volume $V_{4}$

$$
S_{\text {total }} \equiv S_{\text {Density }} \cdot V_{4}=\frac{2 \pi^{2}}{45} \cdot g_{\bullet} \cdot T^{3} \cdot V_{4}
$$

Here the upper value for the temperature is $T \approx 10^{32} \mathrm{~K}$. Further discussions can be seen in [9] as to simple chaotic inflation model, which we view as the template for looking at how to compare this with Padamanadan's treatment of entropy[10] which is with regards to micro canonical ensemble as defined via, if $\Lambda_{M a x}$ is an initial vacuum energy, then by page 505 of [11], formula 10.126 of [11]

$$
\begin{gathered}
\exp \left(S_{\text {total }}\right)=g(E) \leq \frac{A}{N !} \cdot \int d^{3 N} x \cdot[E]^{\frac{3 N}{2}} \approx \\
{\left[\frac{A}{N !} \cdot \int d^{3 N} x\right] \cdot\left[\frac{\Lambda_{M}}{8 \cdot \pi \cdot G} V_{4 x}\right]^{\frac{3 N}{2}}}
\end{gathered}
$$

This gives us the option of comparing what we get in entropy with a quantity similar to what is in [11] again, namely

$$
\frac{\Lambda_{\mathrm{Max}} V_{4}}{8 \cdot \pi \cdot G} \sim T^{00} V_{4} \equiv \rho \cdot V_{4}>>\frac{1}{2} \cdot \sum_{i \neq j} U\left(x_{i}, x_{j}\right)
$$

Where $U$ is a potential energy of self interacting particles in an early universe cosmology. This, when we consider a plasma physics treatment of entropy, we can treat the problem to first order as one of entropy due to an introduction of energy from a prior universe. Note though the time dependence implied by the inter play between equation 12 and equation 13 above. This will have important 
consequences as to treating entropy generation which we will comment upon, [12]later. Reconciling Eq.( 12) and Eq.(13) above though implies as will be commented upon later the enormous value of $\Lambda_{\operatorname{Max}}$ initially which we think is important to understanding how entropy evolved in the first place in a time dependent manner. I.e. the entropy evolved from an initially low pre inflationary value, and arose dramatically during inflation. We will then reference entropy again, via a brane world picture of entropy, and from there come up with a change in entropy expression making use of the idea given above that entropy, which we in this case, is presumably generated by gravitons, as given by Eq.(9) and Eq.(10)is not really a property of say a quark, plasma, or whatever could be modeled by $U$ as a potential energy of self interacting particles in an early universe cosmology. If so then the entropy, if created by a graviton count could be then modeled via initial space-time structures, which we cite below.

\section{Brane World Picture of Early Universe Entropy Formation}

This is adapted from a lecture atICGC-07by Samir Mathur [13]. We propose that branes and anti branes form the working component of an instanton. I.e. we obtain for D space time dimensions, and $\mathrm{E}$ the general energy $S \sim E^{(D-1 / D)}$. Note that Mathur's classical and quantum gravity article [14] has a string winding interpretation of energy along the lines of putting energy $E$ into string windings which leads to an entropy defined in terms of an energy value of, if mass $m_{i}=T_{P} \prod L_{j}$ (with $T_{P}$ being the tension of the $i$ thbrane, and $L_{j}$ being spatial dimensions of a complex torus structure) This leads to entropy

$$
S_{\text {Total }}=A \cdot \prod_{i}^{N} \sqrt{n_{i}}
$$

Our claim is that this very specific value of entropy for Eq. (14) above will at the onset of inflation lead to an early universe entropy count of .

$$
\left|\left[S_{\text {Total }}=A \cdot \prod_{i}^{N} \sqrt{n_{i}}\right] / k_{B} \ln 2\right| \approx[\# \text { operations }]^{3 / 4} \approx 10^{8}(
$$

Note in writing this up the similarity with Lloyd's work[15] Furthermore we claim that the interaction of the branes and anti branes will form an instant on structure, which is implicit in the treatment outlined in Eq. (15), and that the numerical counting given in Eq (14) merely reflects that branes and anti branes, even if charge conjugates of each other have the same 'wrapping number' $n_{i}$. How to tie in the entropy with the growth of the scale function? Racetrack models of inflation, provide a power spectrum given by [16]

$$
P \sim \frac{1}{150 \pi^{2}} \cdot \frac{V(\phi)}{\epsilon}
$$

This is assuming a slow roll parameter treatment with $\in<<1$, and $t>t_{P}$. An increase in scalar power, is then proportional to an increase in entropy via what the author writes as a shift in energy, given by $\Delta E$, as related then to a change in entropy, which we could call

$$
\left|\frac{\Delta E}{l_{P}^{3}}\right| \sim\left|\frac{\Delta P \in 150 \pi^{2}}{l_{P}^{3}}\right| \approx|\Delta S|
$$

Now that we have postulated several specific arguments as to the formulation of entropy, and gravitons and gravitational waves, we will next get to the main point which is the connection with neutrinos and finally the issue which was brought up by Turner [17], [18] which is in the last section of this document. The author saw the material as of the last section of this document in QuyNohn and talked about it with Dr. Turner in Quy Non, which received the author's full attention in August 2013. I.e. how gravitational lensing is connectable with Neutrino physics, and by extension, entropy for the strange CMBR physics noted in Dr. Turner's presentation [18] . To do that, we first review what is said about a non SUSYThis has a non

\section{Neutrino Physics Issues and Changes from the SUSY Paradigm Neutrino.}

K. Meissner and H. Nicolai [19] recently postulated an extension of the SM (standard model) involving a classically conformal Langrangian. The outstanding feature of their model is that if we extend the standard model the way they intend to with the usual Higgs doublet $\Phi$ and one extra weak scalar field $\widetilde{\phi}$ we write as

$$
\widetilde{\phi}(x)=\varphi(x) \cdot \exp \left[\frac{i a(x)}{\sqrt{2} \mu}\right]
$$

Where the field $a(x)$ then gives rise to a (pseudo-) Goldstone particle associated "with the spontaneous breaking of a new global $U(1)_{L}$ (modified Lepton number) symmetry. This boson, the so called 'Majoran'shares many properties with the axion". And furthermore we use conformal symmetry to eliminate in their conformal Lagrangian contributions from all but $\widetilde{\phi}^{4}$ terms in their Lagrangianso eventually we have masses for particles like 'neutrinos' which are heavier than the SUSY neutrino candidate, but with the same "branching ratio " for a particle signature which is like the Higgs but with a lower cross section".

Quoting what was said in the abstract. This ties in with possible new species of detectable neutrinos in ways which lead to an extension of the standard model, since the derived 'axion' is coupled to photons to the tune of $f_{a}=\vartheta\left(10^{15} \mathrm{GeV}\right)$ which is too large for Earthbound experiments but which is in the range of astro physical 
measurements/ experiments, as noted by P. Pugnat et al [20], which could conceivably be detected by proper analysis of the CMBR spectra. If we look at the power spectra as noted in Eq. (16) and its links to fluctuations of entropy (17), we may be able to obtain the necessary conditions for obtaining a signature for how the 'Majoran', i.e. the retro fitted axion, is coupled to photons, while leaving to the LHC determination of actual masses of 'particles' so generated .

Not only would the derived " Axion" be tied into photons, then there would be a way to understand what is otherwise a very puzzling diagram which the author saw in QuyNohn, by Dr. Turner [ 17 ] , [18]

\section{Conclusion}

Now, how does this tie in with the lumpiness seen in the CMBR spectra? Note the following diagram, and this is how GW and Neutrinos may interact. I.e. In QuyNohn, the author interacted with Dr. Turner with the following notes so generated, i.e. Main one transmitted to the author in person was to look at [17], i.e. E---> B "GW" modes implying turbulence in high $\mathrm{L}$ regions of the CMBR spectra, which is part of an interplay between GW lensing and neutrino physics As given by [13] and Turner's presentation in QuyNohn,[18]. His figure 2 is reproduced as Figure 1 for our manuscript. The description of Figure 1 below is altered to fit the renumbering as should be expected.

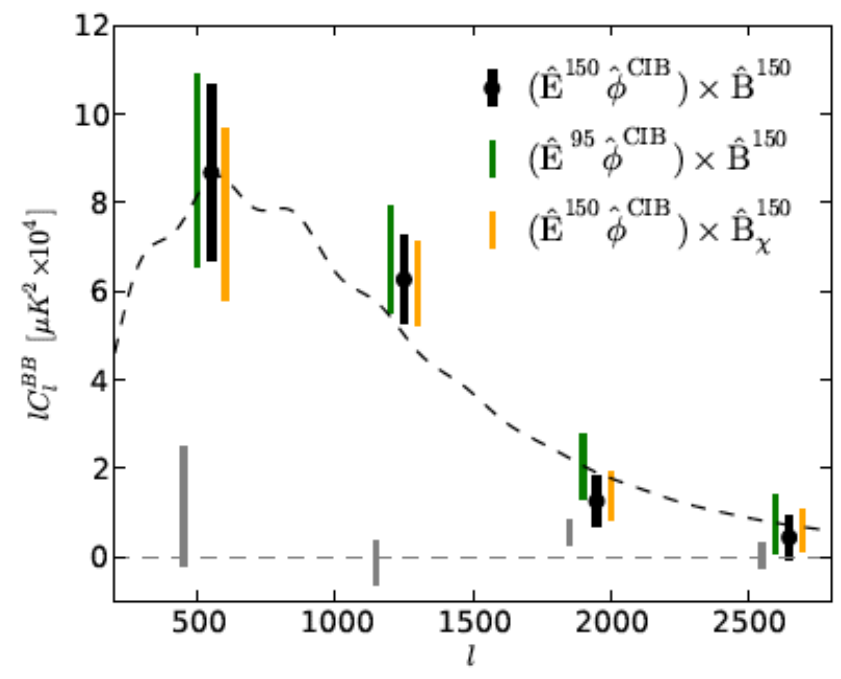

Figure 1. (Black, center bars): Cross-correlation of the lensing B modes measured by SPT pol at $150 \mathrm{GHz}$ with lensing B modes interred from CIB fluctuations measured by Herschel and E modes measured by SPTpol at $150 \mathrm{GHz}$; as shown in a different Figure 1 of reference [18] as well as reference [17] which we denote as having its presence seen in this paper's Figure 1 in the conslucions of this paper. (Green, left-offset bars): Same as black, but using E modes measured at $95 \mathrm{GHz}$, testing both foreground contamination and instrumental systematic. (Orange, right-offset bars): Same as black, but with $\mathrm{B}$ modes obtained using the $\mathrm{X}_{\mathrm{B}}$ procedure described in the text rather than our fiducial Wiener filter. (Gray bars): Curl-mode null test as described in the tex.( Dashed black curve): Lensing B-mode power spectrum in the fiducial cosmological model.

Our discussion as to Figure 1 marks the end of the presentation from the Turner [18] presentation in QuyNhon in Vientam, 2013. So now to conclude, we summarize what can be gleaned as to the lessons which can be inferred from the above manuscript.

In an e mail communication, Subir Sarkar summarized the situation up as follows. "Quasi-DeSitterspacetime during inflation has no "lumpiness" - it is necessarily very smooth. Nevertheless one can generate structure in the spectrum of quantum fluctuations originating from inflation by disturbing the slow-roll of the inflaton - in our model this happens because other fields to which the inflaton couples through gravity undergo symmetry breaking phase transitions as the universe cools during inflation."[21] We intend to examine how this is linkable to entropy variations in Eq. (17) in future numerical simulations of CMBR irregularities. Furthermore, we also should attempt to find traces for the 'axion' photon coupling in careful analysis of the CMBR and use it as part of extending the standard model to accommodate relic early universe neutrino physics without having to invoke SUSY, Technicolor, and some of the other constructions of theoretical physics. We also submit that this model of a non SUSY Neutrino may be optimal to get the CMBR spectra results as seen in [17], [18] which is worthy of future investigation. This idea may also be enhanced by falsification of some of the models as brought up in [22] which the author has read for the last 2 years

\section{REFERENCES}

[1] http://web.ujf-grenoble.fr/HOUCHES/sessions_ete/ete-86/po ster-summer-86.html (see Bouanno)

[2] Alessandra Buonanno, "Gravitational waves" http://arxiv.org/abs/0709.4682, as given by the http://cds.cern.ch/record/1087012; a chapter in Proceedings of Les Houches Summer School, Particle Physics andCosmology: The Fabric of Spacetime, Les Houches, France, 31 Jul - 25 Aug 2006.Les Houches. Session LXXXVI. 2006 ; i.e. Particle Physics and Cosmology: the Fabric of Spacetime, Volume LXXXVI:

[3] Chiu Man Ho, DjordjeMinic, Y. Jack Ng; http://arxiv.org/abs/1201.2365; Ho, Minic and $\mathrm{Ng}$, arXiv:1005.3537[hep-th] (Phys. Lett. B693, 567 (2010))

[4] Y. J. Ng, Spacetime Foam: From Entropy and Holography to Infinite Statistics and Nonlocality, Entropy 2008, 10(4), 441-461; DOI: 10.3390/e10040441.;

[5] Y. Ng,http://cgc.physics.miami.edu/Miami2012/Ng.pdf

[6] A.W. Beckwith, "Is there a generalized way to present entropy?",Prespacetime Journal| May 2011 | Vol. 2 | Issue 5 | pp. 737-742;

[7] A. W. Beckwith, DICE 2010 entry "How to use the cosmological Schwinger principle for energy flux, entropy, and "atoms of space-time" to create a thermodynamic space-time and multiverse", Andrew Beckwith 2011 J. Phys.: 
Conf. Ser. 306012064 doi:10.1088/1742-6596/306/1/01206 4; http://iopscience.iop.org/1742-6596/306/1/012064/

[8] A.W. Beckwith, "Several routes to determining entropy generation in the early universe," arXiv:0712.0029, http://online.itp.ucsb.edu/online/partcosmo_c08/beckwith/

[9] E. W. Kolb and M. S. Turner, The Early Universe, Addison-Wesley, Redwood City, California (1990) [updated paperback edition 1994

[10] Andrew R. Liddle, "The Early Universe", http://arxiv.org/pdf/astro--ph/9612093.pdf

[11] T. Padmanabhan, Cosmology and Astrophysics through Problems, Cambridge University Press, UK, 1996

[12] T. Padmanabhan, Theoretical Astrophysics,Volume 1 : Astrophysical Processes, Cambridge University Press, 2000

[13] SamirMathur ,http://www.iucaa.ernet.in/ icgc07/icgc_plena ry talks/Samir_Mathur.pdf which is fromhttp://www.iucaa.ernet.in/ icgc07/sub/schedule.htm ; http://www.physics.ohio-state.edu/briefcvs/mathur_briefcv.p df

[14] B. D. Chowdhury and S. D. Mathur; "Fractional brane state in the early universe" Class. Quant. Grav. 24, 2689 (2007) [arXiv:hep-th/0611330]
[15] Seth Lloyd, "Computational capacity of the Universe", http://arxiv.org/abs/quant-ph/0110141, Phys.Rev.Lett.88:237901,2002

[16] PhBrax, A C Davis, S C Davis,RJeannerot, and, M. Postma,"D-term Uplifted Racetrack Inflation", http://arxiv.or $\mathrm{g} / \mathrm{pdf} / 0710.4876 . \mathrm{pdf}$

[17] D. Hansen,et.al. "Detection of B-mode Polarization in the Cosmic Microwave Background with Data from the South Pole Telescope"http://arxiv.org/abs/1307.5830

[18] .S.Turner, http://vietnam.in2p3.fr/2013/Inauguration/http://vietnam.in2 p3.fr/2013/Inauguration/program.htmlgo to ; Review of the standard model of Cosmology, S. Turner ( August 12)

[19] K.A. Meissner and H. Nicolai, “ Neutrinos, Axions, and Conformal Symmetry”, arXiv: 0803.2814v2, 2 April 2008

[20] P. Pugnat et al., "First results from the OSQAR photon regeneration experiment: No light shining through a wall" arXiv:0712.3362 [ hep-ex ]

[21] Subir Sarkar, private e mail to the Author, February 22, 2008

[22] MaurioGasperni,"Elements of String Cosmology", Cambridge University press, New York City, NY, USA, 2007 